\title{
On a question of Bourgain about geometric incidences*
}

\author{
József Solymosi ${ }^{\dagger} \quad$ Csaba D. Tóth ${ }^{\ddagger}$
}

\begin{abstract}
Given a set of $s$ points and a set of $n^{2}$ lines in three-dimensional Euclidean space such that each line is incident to $n$ points but no $n$ lines are coplanar, then we have $s=\Omega\left(n^{11 / 4}\right)$. This is the first nontrivial answer to a question recently posed by Jean Bourgain.
\end{abstract}

\section{Introduction}

A seminal result in geometric incidences is the Szemerédi-Trotter bound [24, which says that the number of incidences between $n$ points and $m$ lines in the Euclidean plane is at most $O\left(n^{2 / 3} m^{2 / 3}+\right.$ $n+m)$, and it is the best possible. Typical problems in geometric incidences consider two families of geometric objects of size $n$ and $m$, respectively, and asks for the maximum number of incident pairs. In three and higher dimensions, non-trivial bounds often require restrictions on the geometric objects, otherwise every object of one family might be incident to all objects of the other family, or the number of incidences might be maximized when all objects lie in a lower dimensional affine subspace.

In the 1990-s, Tom Wolff [27, 26] observed that tight bounds on the number of incidences can be used efficiently to attack problems related to the Kakeya conjecture, one of the central conjectures in harmonic analysis. For more details about the Kakeya conjecture, refer to [4] or [26]. Bennett, Carbery, and Tao [3] established a concrete connection between multilinear Kakeya estimates and bonds on number of incidences between points and lines in three dimensions.

Recently, Jean Bourgain [10] asked what is the minimum cardinality $s$ of a point set $S$ in threedimensional Euclidean space, if we are given $n^{2}$ lines, each of which1 is incident to $n$ points of $S$ but no $n$ lines are coplanar. He conjectured that $s=\Omega\left(n^{3-\varepsilon}\right)$ for every constant $\varepsilon>0$. If the $n^{2}$ lines are disjoint, then $s=n^{3}$ is obvious. The integer grid $\left\{(a, b, c) \in \mathbb{R}^{3}: 1 \leq a, b, c \leq n\right\}$ and the set of $3 n^{2}$ axis-aligned lines also gives $s=n^{3}$ points. The Szemerédi-Trotter theorem, applied to $n^{2}$ lines and $s$ points with $n^{3}$ point-line incidences, gives a lower bound of $s=\Omega\left(n^{5 / 2}\right)$. This bound, however, does not use the condition that no $n$ lines are coplanar. We give the first nontrivial answer to Bourgain's question.

Theorem 1 Given a set of $s$ points and a set of $n^{2}$ lines in $\mathbb{R}^{3}$ such that every line is incident to at least $n$ points but no $n$ lines are coplanar, we have $s=\Omega\left(n^{11 / 4}\right)=\Omega\left(n^{3-\frac{1}{4}}\right)$.

\footnotetext{
${ }^{*}$ The research was partially supported by OTKA and NSERC grants. The first author was supported by a Sloan Research Fellowship.

${ }^{\dagger}$ Department of Mathematics, University of British Columbia, Vancouver, BC, Canada, solymosi@math.ubc.ca

${ }^{\ddagger}$ Department of Mathematics, Room 2-336, MIT, Cambridge, MA 02139, USA, toth@math.mit.edu
} 
Related previous work. The Szemerédi-Trotter bound on the number of point-line incidences is tight in the plane: $n$ points and $m$ lines in the plane may have $\Omega\left(n^{2 / 3} m^{2 / 3}+n+m\right)$ incidences. A better upper bound in three-dimensions is possible only under certain restrictions, which guarantee that the given point-line configuration is "far" from being planar. Bourgain's condition that no $n$ of $n^{2}$ given lines are coplanar is one such restriction. Another previously considered condition requires every point to be incident to at least three non-coplanar lines, such a point is called joint. Sharir [20] conjectured that the number of joints for $m$ lines in $\mathbb{R}^{3}$ is $O\left(m^{3 / 2}\right)$, which is attained by the axis-aligned lines of the $\sqrt{m} \times \sqrt{m} \times \sqrt{m}$ integer lattice section. The best current bound, $O\left(m^{1.6232}\right)$ by Feldman and Sharir [14, is far from being tight. Sharir and Welzl [21] gave an $O\left(\mathrm{~m}^{5 / 3}\right)$ upper bound on the number of joint-line incidences in three-space.

Edelsbrunner et al. [11] were the first to study the number of point-plane incidences in three dimensions. They obtained an $O\left(n^{3 / 5} m^{4 / 5}+n+m \log n\right)$ bound for $n$ points and $m$ planes assuming that no three points are collinear. Note that $n$ points and $m$ planes in three-space may have $n m$ incidences if the points are collinear and all $m$ planes contain this line. Every non-trivial bound on point-plane incidences must, therefore, impose some reasonable restriction. Agarwal and Aronov [1] proved an upper bound of $O\left(\mathrm{~nm}^{2 / 3}+n^{2}\right)$ for point-plane incidences in $\mathbb{R}^{3}$ assuming that each plane is spanned by the point set (that is, each plane contains three affine independent points). Their bound matches the lower bound of Edelsbrunner and Haussler [12]. Braß and Knauer [6] gave an $O\left(n^{3 / 4} m^{3 / 4} \log (n m)+(n+m) \log (n+m)\right)$ bound assuming that the incidence graph does not contain a $K_{r, r}$ for some fixed $r \in \mathbb{N}$. Elekes and Tóth [13] obtained a tight bound of $O\left(n^{3 / 4} m^{3 / 4}+n \sqrt{m}+n\right)$ for the incidences between $n$ points and $m$ saturated planes (where a plane is called saturated if at most a constant fraction of the points lying in the plane are collinear). It is attained by a system where all points lie in two parallel planes. Solymosi and Tóth [22] gave an $O\left(n^{3 / 4} m^{3 / 4}\right)$ bound for homogeneous point sets, which covers the example of the integer lattice section. Solymosi and $\mathrm{Vu} 23$ shoed that if $S$ is a homogeneous set of $n$ points in three-space and $k \geq 2$, then the number of $k$-rich lines is at most $O\left(n^{2} / k^{4}\right)$. Note that this result implies Bourgain's conjecture for homogeneous point sets.

Proof techniques and Organization. Essentially two different methods have been developed for proving geometric incidence bounds: One is the crossing number technique based on work by Székely [25]; the other is the $\varepsilon$-cutting technique, which is a divide-and-conquer strategy introduced by Clarkson and Shor [9], and some tight bounds were obtained by Chazelle and Friedman [8] (see also, [7, 17]). We deploy both techniques. Refer to a survey by Pach and Sharir [18] for the rich history and widespread applications of these techniques.

In Section 2, we use ideas of Sharir and Welzl 21] to represent lines meeting a given line and doubly ruled surfaces by points and algebraic curves in the plane; and then we apply the crossing technique in the plane. In Section 3, we apply the cutting technique to reduce the problem to the case that every point is incident to $\Omega(1)$ lines on average, and complete the proof of Theorem 1 with an extremal graph theoretical bound on the number of incidences of lines and doubly ruled surfaces.

\section{Lines and reguli in three-space}

A regulus is a doubly ruled quadratic surface in three-space [15]. Every regulus contains two families of lines, which are called rulings: Each ruling consists of pairwise skew lines, and every line of one 
ruling intersects all the lines of the other ruling of a regulus. We say that every line of each ruling is incident to the regulus. Any three pairwise skew lines are contained in a ruling of a unique regulus. If a line has three common points with a regulus, then it must be incident to that regulus (furthermore, it is part of one ruling of that regulus).

Since no three lines are incident to two distinct rulings, we can apply the Kővári-Sós-Turán bound from extremal graph theory [16]: A bipartite graph with $m$ and $r$ vertices in its two vertex classes and having no subgraph isomorphic to $K_{3,2}$ has at most $O\left(m r^{2 / 3}+r\right)$ edges. It follows that the number of line-regulus incidences between $m$ lines and $r$ reguli in three-space is bounded by $O\left(m r^{2 / 3}+r\right)$.

We extend two lemmas of Sharir and Welzl [21] on the number of point-regulus incidences. Both concern the number of reguli incident to a given line $\ell$, and spanned by three lines of a given set of lines $M$. The first lemma gives an upper bound on the number line-reguli incidences; the second lemma gives a lower bound on the the number of reguli under the condition that at most $|M| / 5$ lines of $M$ may be coplanar or concurrent.

Lemma 2 Assume that a line $\ell$ meets every element of a set $M$ of $m$ lines in three-space and we are given a set $R$ of $r$ reguli. The number of incidences between $R$ and $M$ is bounded by $O\left(m^{3 / 5} r^{4 / 5}+m+r\right)$.

Proof. If a regulus $\varrho \in R$ is incident to two intersecting lines of $M$, it cannot be incident to line $\ell$. Hence such a $\varrho$ intersects $\ell$ in at most two points, and so it contains at most four lines of $M$. The reguli in $R$ that are incident to up to four lines in $M$ are responsible for at most $4 r$ incidences. Let $Q$ denote the set of reguli in $R$ incident to at least three pairwise skew lines of $M$. We can represent the lines that meet $\ell$ by points in three-space: For instance, one dimension can be the intersection with $\ell$, and two additional dimensions can be the coordinates of their intersection point with a plane parallel to $\ell$. The families of lines incident to reguli spanned by $M$ correspond to bounded degree algebraic curves in three-space [21].

Project these points and curves to a generic plane. We obtain a set $P$ of $m$ points and a set $C$ of at most $r$ bounded degree algebraic curves such that any three points of $P$ are incident to at most one curve of $C$. By result of Pach and Sharir [19], the number of point-curve incidences is bounded by $O\left(m^{3 / 5} r^{4 / 5}+m+r\right)$. Together with $O(r)$ incidences of the reguli in $R \backslash Q$, there are $O\left(m^{3 / 5} r^{4 / 5}+m+r\right)$ line-regulus incidences between $M$ and $R$.

Remark: Lemma 2 is not sharp. With a little work, one could show that the curves in the plane are pseudo-parabolas, for which a better incidence bound is available 2]. Using this bound, one could show that there are at most $O\left(m^{6 / 11-\epsilon} r^{9 / 11}+m+r\right)$ line-regulus incidences between $M$ and $R$. This bound, however, is not a bottle neck in our estimates, and the bound of Lemma 2 suffices for our purposes.

Lemma 3 Assume that a line $\ell$ meets every element of a set $M$ of $m$ lines in three-space such that at most $m / 5$ lines of $M$ may be coplanar or concurrent. Then $\ell$ is incident to at least $\Omega\left(m^{3}\right)$ distinct reguli spanned by $M$.

Proof. It is easy to see that there are at least $\mathrm{m}^{3} / 50$ (unordered) triples of pairwise skew lines in $M$. First notice that there are at least $m(3 m / 5)(m / 5)=3 m^{3} / 25$ ordered triples $\left(\ell_{1}, \ell_{2}, \ell_{3}\right) \in M^{3}$ of pairwise skew lines in $M$ : Choose any line $\ell_{1} \in M$; then choose any line $\ell_{2} \in M$ that is not incident to the point $\ell \cap \ell_{1}$ and does not lie in the plane $\pi\left(\ell, \ell_{1}\right)$ spanned by $\ell$ and $\ell_{1}$ (there are at 
least $3 \mathrm{~m} / 5$ such lines); finally choose any line $\ell_{3} \in M$ that is not incident to $\ell \cap \ell_{1}$ or $\ell \cap \ell_{2}$ and does not lie in the planes $\pi\left(\ell, \ell_{1}\right)$ or $\pi\left(\ell, \ell_{2}\right)$.

These $m^{3} / 50$ triples of pairwise skew lines do not necessarily span distinct reguli, but we show that only few reguli can be incident to too many lines of $M$. For every $t \in \mathbb{N}$, let $R_{t}$ denote the set of reguli spanned by $M$ and incident to at least $t$ lines of $M$. There are at least $t\left|R_{t}\right|$ incidences on these reguli, and by Lemma 2, this number is bounded by $O\left(m^{3 / 5}\left|R_{t}\right|^{4 / 5}+m+\left|R_{t}\right|\right)$. It follows that $\left|R_{t}\right| \leq O\left(m^{3} / t^{5}+m / t\right)$. Set $t$ to be a large constant such that $\left|R_{t}\right| \leq m^{3} / 100$. It follows that at least $m^{3} / 100$ triples of $M$ span reguli, each incident to less than $t$ lines. Hence $M$ spans at least $m^{3} /\left(100\left(\begin{array}{l}t \\ 3\end{array}\right)\right)=\Omega\left(m^{3}\right)$ distinct reguli.

\section{Proof of the main theorem}

We are given a set $L$ of $n^{2}$ lines and a set $S_{0}$ of points in $\mathbb{R}^{3}$ such that every line in $L$ is incident to $n$ points and no $n$ lines are coplanar. There are $n^{3}$ point-line incidences, where each incidence is a pair $(p, \ell) \in S_{0} \times L$ with $p \in \ell$. Let $S$ be the set of points in $S_{0}$ incident to at most $n$ lines of $L$ and set $s=|S|$. By the Szemerédi-Trotter theorem, at most $O\left(|L|^{2} / n^{3}+|L| / n\right)=O(n)$ lines of $S_{0}$ are incident to $n$ or more lines, and these points are involved in at most $O\left(n^{2} \log n\right)$ incidences. If $n$ is sufficiently large, then the remaining $s$ points in $S$ and $n^{2}$ lines in $L$ still have at least $n^{3} / 2$ incidences, furthermore, no $n$ lines of $L$ are coplanar or meet at a point of $S$. Let $d=n^{3} / s$ denote the average number of lines incident to a point of $S$.

Project the lines of $L$ and the points of $S$ into a generic plane, and consider the dual arrangement. We obtain a set $L^{*}$ of $n^{2}$ points and a set $S^{*}$ of $s$ lines in the plane such that every point in $L^{*}$ is incident to at most $n$ lines, every line in $S^{*}$ is incident to at most $n$ points, and there are at least $n^{3} / 2$ point-line incidences. Choose a parameter $r=c d$ with a sufficiently small constant $c>0$ to be specified by two upper bounds below. Consider a $(1 / r)$-cutting [ 8 ] for $S^{*}$, which is a partition of the plane into $O\left(r^{2}\right)$ triangles such that the interior of each triangle intersects at most $s / r$ lines of $S^{*}$. By splitting some triangles, if necessary, we obtain a partition of the plane into a set $\Xi$ of $O\left(r^{2}\right)$ triangles, each containing at most $n^{2} / r^{2}$ points of $L^{*}$. For every triangle $\sigma \in \Xi$, let $L_{\sigma}^{*}$ denote the set of points of $L^{*}$ in $\sigma$, and let $S_{\sigma}^{*}$ be the set of lines of $S^{*}$ intersecting the interior of $\sigma$.

The number of point-line incidences involving points on the boundary of some triangles and lines intersecting the interior of an adjacent triangle is bounded by $I_{\text {boundary }}=O\left(r^{2}\right) \cdot(s / r)=O(s r)=$ $O(c s d)=O\left(c n^{3}\right)$. Let $c>0$ be so small that $I_{\text {boundary }}<n^{3} / 2$. Hence the sum of incidences in each triangle is at least $n^{3} / 4$, that is,

$$
\sum_{\sigma \in \Xi} I\left(L_{\sigma}^{*}, S_{\sigma}^{*}\right) \geq \frac{n^{3}}{4} .
$$

There is a triangle $\sigma$ such that $I\left(L_{\sigma}^{*}, S_{\sigma}^{*}\right) \geq\left(n^{3} / 4\right) /|\Xi|=\Omega\left(n^{3} /\left(c^{2} d^{2}\right)\right)$. Let $L_{\sigma} \subset L$ and $S_{\sigma} \subset S$ denote, respectively, the lines and points corresponding to planar duals of $L_{\sigma}^{*}$ and $S_{\sigma}^{*}$. We have a set $L_{\sigma}$ of at most $O\left(n^{2} /\left(c^{2} d^{2}\right)\right)$ lines and a set $S_{\sigma}$ of at most $s /(c d)$ points in three-space that have at least $\Omega\left(n^{3} /\left(c^{2} d^{2}\right)\right)$ incidences.

We next give a lower bound on the number of line pairs

$$
G=\left\{\left(\ell_{1}, \ell_{2}\right) \in L_{\sigma}: \ell_{1} \cap \ell_{2} \in S_{\sigma}\right\}
$$

that meet at a point of $S_{\sigma}$. Denoting by $d_{\sigma}(p)$ the number of lines of $L_{\sigma}$ incident to a point $p \in S_{\sigma}$, 
we have

$$
|G|=\sum_{p \in S_{\sigma}}\left(\begin{array}{c}
d_{p} \\
2
\end{array}\right) \geq\left|S_{\sigma}\right| \cdot\left(\begin{array}{c}
\left(\sum_{p \in S_{\sigma}} d_{p}\right) /\left|S_{\sigma}\right| \\
2
\end{array}\right) .
$$

We can estimate the average degree by

$$
\frac{\sum_{p \in S_{\sigma}} d_{p}}{\left|S_{\sigma}\right|}=\frac{I\left(S_{\sigma}, L_{\sigma}\right)}{\left|S_{\sigma}\right|} \geq \Omega\left(\frac{n^{3}}{c d s}\right)=\Omega\left(\frac{1}{c}\right) .
$$

Hence, at least $|G|=\Omega\left(\left|S_{\sigma}\right| / c^{2}\right)=\Omega\left(s /\left(c^{3} d\right)\right)$ line pairs meet at points of $S_{\sigma}$. Discard all lines $\ell \in L_{\sigma}$ that meet less than $5 n$ other lines of $L_{\sigma}$ at points of $S_{\sigma}$. We have discarded at most $5 n\left|L_{\sigma}\right|=O\left(n^{3} /\left(c^{2} d^{2}\right)\right)=O\left(s /\left(c^{2} d\right)\right)$ line pairs. Set the constant $c>0$ so small that we discard at most $|G| / 2$ line pairs of $G$. In the remainder of the proof, $c$ is fixed and hidden in the asymptotic notation.

We have a set $L_{\sigma}^{\prime}$ of $O\left(n^{2} / d^{2}\right)=O\left(n^{2} /\left(n^{3} / s\right)^{2}\right)=O\left(s^{2} / n^{4}\right)$ lines such that each line meets at least $5 n$ other lines of $L_{\sigma}^{\prime}$ and the total number of meeting pairs of lines is at least $\Omega(s / d)=$ $\Omega\left(s^{2} / n^{3}\right)$. Recall that no $n$ lines of $L$ are coplanar or meet at a point of $S$. Let $R_{\sigma}^{\prime}$ denote the set of reguli spanned by lines of $L_{\sigma}^{\prime}$. By Lemma 3, a line $\ell \in L_{\sigma}^{\prime}$ that meets $m_{\ell}$ other lines of $L_{\sigma}^{\prime}$, where $m_{\ell} \geq 5 n$, is incident to at least $\Omega\left(m_{\ell}^{3}\right)$ reguli of $R_{\sigma}^{\prime}$. The total number of line-reguli incidences in $L_{\sigma}^{\prime} \times R_{\sigma}^{\prime}$ is bounded from below by

$$
\begin{aligned}
I\left(L_{\sigma}^{\prime}, R_{\sigma}^{\prime}\right) & =\sum_{\ell \in L_{\sigma}^{\prime}} \Omega\left(m_{\ell}^{3}\right) \geq\left|L_{\sigma}^{\prime}\right| \cdot \Omega\left(\left(\frac{\sum_{\ell \in L_{\sigma}^{\prime}} m_{\ell}}{\left|L_{\sigma}^{\prime}\right|}\right)^{3}\right)=\Omega\left(\frac{1}{\left|L_{\sigma}^{\prime}\right|^{2}} \cdot\left(\sum_{\ell \in L_{\sigma}^{\prime}} m_{\ell}\right)^{3}\right) \\
& =\Omega\left(\frac{\left(s^{2} / n^{3}\right)^{3}}{\left(s^{2} / n^{4}\right)^{2}}\right)=\Omega\left(\frac{s^{2}}{n}\right) .
\end{aligned}
$$

On the other hand, $\left|L_{\sigma}^{\prime}\right|=O\left(s^{2} / n^{4}\right)$ lines can span at most

$$
\left(\begin{array}{c}
\left|L_{\sigma}^{\prime}\right| \\
3
\end{array}\right)=O\left(\frac{s^{6}}{n^{12}}\right)
$$

reguli. By the Kővari-Sós-Turán bound, the number of line-regulus incidences is bounded by

$$
I\left(L_{\sigma}^{\prime}, R_{\sigma}^{\prime}\right)=\Omega\left(\left|L_{\sigma}^{\prime}\right| \cdot\left|R_{\sigma}^{\prime}\right|^{2 / 3}+\left|R_{\sigma}^{\prime}\right|\right)=O\left(\left|L_{\sigma}^{\prime}\right|^{3}\right)=O\left(\frac{s^{6}}{n^{12}}\right) .
$$

Comparing the upper and lower bounds on the number of line-regulus incidences $I\left(L_{\sigma}^{\prime}, R_{\sigma}^{\prime}\right)$, we have

$$
\Omega\left(\frac{s^{2}}{n}\right) \leq I\left(L_{\sigma}^{\prime}, R_{\sigma}^{\prime}\right) \leq O\left(\frac{s^{6}}{n^{12}}\right),
$$

that is, $s=\Omega\left(n^{11 / 4}\right)$, as required.

\section{References}

[1] P. K. Agarwal and B. Aronov, Counting facets and incidences, Discrete Comput. Geom. 7 (1992), 359-369. 
[2] P. Agarwal, E. Nevo, J. Pach, R. Pinchasi, M. Sharir and S. Smorodinsky, Lenses in arrangements of pseudocircles and their applications, J. ACM 51 (2004), 139-186.

[3] J. Bennett, A. Carbery, and T. Tao, On the multilinear restriction and Kakeya conjectures, Acta Mathematica 196 (2) (2006), 261-302.

[4] J. Bourgain, On the dimension of Kayela sets and related maximal inequalities, Geom. Funct. Anal. 9 (1999), 256-282.

[5] J. Bourgain, N. Katz, T. Tao, A sum-product estimate in finite fields, and applications, Geom. Funct. Anal. 14 (1) (2004), 27-57.

[6] P. Braß and C. Knauer, On counting point-hyperplane incidences, Comput. Geom. Theory Appl. 25 (2003), 13-20.

[7] B. Chazelle, Cuttings, in Handbook of Data Structures and Applications, CRC Press, Boca Raton, FL, 2005, pp. 1-25,.

[8] B. Chazelle and J. Friedman, A deterministic view of random sampling and its use in geometry, Combinatorica 10 (3)(1990), 229-249.

[9] K. L. Clarkson and P. W. Shor, Applications of random sampling in computational geometry. II. Discrete Comput. Geom. 4 (1989), 387-421.

[10] E. Croot and V. F. Lev, Problems presented at the Workshop on Recent Trends in Additive Combinatorics, American Institute of Mathematics, Palo Alto, CA, 2004.

[11] H. Edelsbrunner, L. J. Guibas, and M. Sharir, The complexity of many cells in arrangements of planes and related problems, Discrete Comput. Geom. 5 (1990), 197-216.

[12] H. Edelsbrunner and D. Haussler, The complexity of cells in three-dimensional arrangements, Discrete Math. 60 (1986), 139-146.

[13] Gy. Elekes and Cs. D. Tóth, Incidences of not-too-degenerate hyperplanes, in Proc. 21st ACM Sympos. Comput. Geom., ACM Press, 2005, pp. 16-21.

[14] S. Feldman and M. Sharir, An improved bound for joints in arrangements of lines in space, Discrete Comput. Geom. 33 (2005), 307-320.

[15] D. Hilbert, Geometry and the Imagination, Chelsea. Pub. Co., New York, 1952.

[16] T. Kővari, V. T. Sós, and P. Turán, On a problem of K. Zarankiewicz, Colloquium Math. 3 (1954), 50-57.

[17] J. Matoušek, Lectures on Discrete Geometry, Springer-Verlag, Berlin, 2002.

[18] J. Pach and M. Sharir, Geometric incidences, in Towards a theory of geometric graphs, vol. 342 of Contemp. Math., AMS, Providence, RI, 2004, pp. 185-223.

[19] J. Pach and M. Sharir, On the number of incidences between points and curves, Combinatorics, Probability $\&$ Computing 7 (1998), 121-127.

[20] M. Sharir, On joints in arrangements of lines in space and related problems, J. Combin. Theory Ser. A 67 (1994), 89-99.

[21] M. Sharir and E. Welzl, Point-line incidences in space, Combinatorics, Probability \& Computing 13 (2004), 203-220.

[22] J. Solymosi and Cs. D. Tóth, Distinct distances in homogeneous sets in euclidean space, Discrete Comput. Geom. 35 (4) (2006), 537-549.

[23] J. Solymosi and V. H. Vu, Distinct distances in homogeneous sets, Proc. 19th Sympos. on Comput. Geom., ACM Press, 2003, pp. 104-105. 
[24] E. Szemerédi and W. T. Trotter Jr., Extremal problems in Discrete Geometry, Combinatorica 3 (3-4) (1983), 381-392.

[25] L. A. Székely, Crossing numbers and hard Erdős problems in discrete geometry, Combinatorics, Probability \& Computing 6 (3) (1997), 353-358.

[26] T. H. Wolff, Lectures in Harmonic Analysis (I. Laba and C. Shubin, eds.), vol 29 of University Lecture Series, AMS, Providence, RI, 2003.

[27] T. H. Wolff, Recent work connected with the Kakeya problem, in Prospects in Mathematics: Invited Talks on the Occasion of the 250th Anniversary of Princeton University (H. Rossi, ed.), 129-162, AMS, Providence, RI, 1999. 